\title{
An Improved Zero-Forcing Detection Algorithm of Spread OFDM for Underwater Acoustic Communications
}

\author{
Ke WANG \\ College of Electronic Science and Engineering \\ National University of Defense Technology \\ Changsha, Hunan Province, China \\ e-mail: wangke11a@nudt.edu.cn \\ Wei LIU \\ College of Electronic Science and Engineering \\ National University of Defense Technology \\ Changsha, Hunan Province, China \\ e-mail: wliu_nudt@nudt.edu.cn
}

\author{
Leiyu SHI \\ The Information Support Base \\ Beijing, China \\ e-mail: leiyu_shi@163.com
}

\author{
Jing LEI \\ College of Electronic Science and Engineering \\ National University of Defense Technology \\ Changsha, Hunan Province, China \\ e-mail: leijing@nudt.edu.cn
}

\begin{abstract}
The so-called spread OFDM(S-OFDM) system is designed to resist frequency-dependent attenuation by using Walsh-Hadamard (WH) code for spreading the frequency-domain OFDM symbols in the transmitter, and linear detectors, like ZF or MMSE, are usually used to recover the signals in the receiver. In this paper, an improved ZF detection algorithm is proposed for reducing the problem of amplifying noise compared with common ZF algorithm for spread OFDM in underwater acoustic channels. In contrast to the MMSE, the proposed algorithm dose not require estimating the variance of noise. Simulation results show that the proposed ZF algorithm has much better performance than that of common ZF method in all SNR range. Even if compared with MMSE, the proposed algorithm also gets much performance gain in high SNR range.
\end{abstract}

Keywords-OFDM; underwater acoustic communication; Walsh-Hadamard (WH); zero force; minimum mean square error

\section{INTRODUCTION}

Generally, underwater acoustic communications has poor link quality due to the multipath and time variability of underwater channels. The main cause for multipath propagation is the reflections from surface and bed of ocean, it produces big delay to the underwater acoustic signal and then causes severe frequency-dependent attenuation [1,2,3].

To resist the excess delay spread inherited in underwater acoustic channel, multicarrier communication is an alternative for its long symbol duration. Orthogonal frequency division multiplexing (OFDM) transmits signals over multiple orthogonal subcarriers simultaneously, it can obtain outstanding performance in tough multi-path environments, achieving reasonable spectral efficiency and data rate [4,5]. Despite its remarkable adaptation to multipath channel, one of the main concerns in OFDM is the loss of multipath diversity. The reason is that each frequency-domain symbol is transmitted over a flat sub-channel individually, so that it will provide no guarantees in correctly detection if the corresponding sub-channel falls deeply fading.

There have been several proposals in the literature to alleviate the problem of deeply fading for OFDM. One of the existing techniques, which has received amount of research attention, is based on spreading each data symbol across all OFDM subcarriers [6,7], so that each subcarrier contains a linear combination of all the data symbols. Then, the deeply degraded subcarriers can also be recovered through a de-spread process in the receiver. This spreading is usually done by a pre-coding unitary matrix (e.g. the Walsh-Hadamard [8]). Currently, such spread OFDM technique has been introduced into underwater acoustic communications in $[9,10]$ for resisting the strong multipath in underwater acoustic channels. Rather than maximum likelihood (ML) receiver, whose complexity would be exponential growth with the number of symbols spread, the simple linear receivers, like zero-forcing (ZF) and minimum mean square error (MMSE), are often used in S-OFDM[7,11]. However, the ZF detector has the disadvantage of amplifying noise; the MMSE detector, which cuts down amplifying noise, has to estimate the variance of noise [6].

In this paper, we propose an improved ZF detecting algorithm for S-OFDM with underwater acoustic channels, where those sub-carriers with the channel power below a predesigned threshold will be deleted before de-spreading and ZF detecting in order to avoid amplifying noise. The data in the deleted sub-carriers can still be recovered since other sub-carriers remained also include their data due to spreading process. By using such a simple sub-carrier deleting method, the proposed ZF obtains much better performance than that of common ZF, and also outperforms the MMSE in high SNR range, which will be certified by simulations.

The remaining part of the paper is organized as follows: Sect. 2 presents the basics of OFDM system and the concept of S-OFDM. Sect. 3 presents the different detectors. Section 4 introduces the proposed ZF detector. Sect. 5 discusses the 
channel model, simulation parameters and showing the simulation results. Sect. 6 concludes the paper.

\section{S-OFDM SYSTEM}

In conventional OFDM, the transmitted data are split into $N$ parallel streams, then go through an $N$ point IFFT operation to produce a complex OFDM signal:

$$
x[m]=\frac{1}{2 N} \sum_{n=0}^{N-1} s[n] e^{j \frac{2 \pi}{N} m}
$$

Note that $x[m]$ is the $m$ th time domain sample of OFDM symbol. $s[n]$ is the frequency domain symbol to be transmitted on the $n$th subcarrier.

In a frequency selective underwater channel, there always exits deeply faded subchannels, which will distort the OFDM subcarriers that are in their neighborhood, resulting in a loss of symbols carried by these subcarriers. And this ultimately results in a high BER. The approach we used is to scatter data symbols over the entire bandwidth. This technique is called spread OFDM. Fig.1 is the system model of S-OFDM. The every single symbol is spread into all subcarriers. So if some subcarriers are faded intensely, we can also use simple linear matrix operation to recover all data symbols in the receiver.

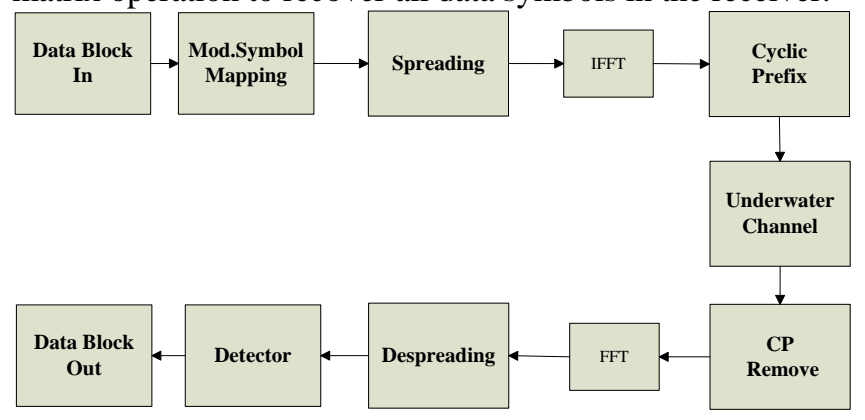

Figure 1. System model of S-OFDM

The spread matrix chosen in this paper is Walsh-Hadamard matrix. The Walsh-Hadamard Transform(WHT) for a vector with length of $2 \times m$ can be achieved by multiplying a $2 m \times 2 m$ matrix:

$$
W_{2 m}=\left[\begin{array}{cc}
W_{m} & W_{m} \\
W_{m} & -W_{m}
\end{array}\right]
$$

Where $W_{1}= \pm 1$, then:

$$
W_{2}=\left[\begin{array}{ll}
W_{1} & W_{1} \\
W_{1} & -W_{1}
\end{array}\right]=\left[\begin{array}{cc}
+1 & +1 \\
+1 & -1
\end{array}\right]
$$

Then we can get any $2 m \times 2 m$ WH matrix, the WHT of a symbol vector can be represented as:

$$
\overrightarrow{S_{W}}=\frac{W_{N}}{\sqrt{N}} \times \vec{s}[n]
$$

$N$ is the length of $\vec{s}[n]$, the purpose of normalization is to keep the energy of the vector unchanged. For WH matrix, the coefficient is always \pm 1 , so after IFFT, the PAPR of the vector $S_{W}[n]$ will be lower compared to conventional OFDM.

In receiver, inverse WH Transform(IWHT) can be achieved by multiplying a normalized WH matrix $W_{N}$, i.e.

$$
\vec{S}=\frac{W_{N}}{\sqrt{N}} \times \overrightarrow{S_{W}}
$$

S-OFDM is a simply modified OFDM compared to the conventional OFDM model. In transmitter, after a spread operation, the spread frequency domain signal is fed to IFFT:

$$
x=F^{H} C S
$$

Note that $S$ is transmitted symbol vector with length $N$. $C$ is the normalized spread matrix, in this paper, we choose WH spread, so $C=W_{N} / \sqrt{N}$. For the conventional OFDM model with no spreading, $C$ is simply the $N \times N$ identity matrix $I_{N} \cdot F^{H}$ is the $N$ point normalized IFFT matrix.

The entire transmit process is depicted in Fig.1, we assume there are $L$ paths in a frequency selective underwater channel, every path is represented by a complex zero-mean Rayleigh distributed gain $h_{l}$ with unity variance:

$$
\mathrm{h}=\left[h_{0}, h_{1}, \cdots, h_{L-1}\right]
$$

Then after OFDM demodulation, the received signal can be represented:

$$
\mathrm{y}=G C s+F v
$$

$$
G=F C_{p r} H C_{p} F^{H}
$$

Note that $v$ is $N \times 1$ AWGN noise vector with variance matrix $\sigma_{v}^{2} I_{N}$. And multiplying $F$ in the receiver doesn't change its variance. $F$ is the normalized FFT matrix used for 
OFDM demodulation. $C_{p}$ and $C_{p r}$ are cyclic prefix and cyclic prefix removal operators respectively. $H$ is the convolution matrix of channel vector $h$. According to the property of cyclic prefix, the multiplication $C_{p r} H C_{p}$ results in a circulant matrix where each column is a circular shift by one relative to the previous column, with the first column formed from the zero-padded impulse response of the channel vector $h$. For the reason that the subcarriers in OFDM are orthogonal, $G$ in Eq.9 becomes a diagonal matrix. The diagonal entries of $G$ are the $N$ point DFT values of the channel vector $h$, meanwhile they are the channel response for every individual subcarrier.

\section{S-OFDM DETECTION}

We will introduce two detection techniques for S-OFDM system namely ZF and MMSE. For the enormous calculate complexity, we don't consider the Maximum Likelihood (ML) algorithm. Assuming the receiver can get perfect channel information, i.e. the matrix $G$.

A. $Z F$

The way of ZF detector is to remove the interference of channel by multiplying $G^{-1}$ to the received signal $y$ directly, then we can get the received symbol:

$$
\hat{S}_{\text {SOFDM }}=(G C)^{-1} y
$$

For the conventional OFDM with no spread, $C=I_{N}$, then the detected symbol is:

$$
\hat{S}_{\text {SOFDM }}=G^{-1} y
$$

From sect.2 we know that the equivalent frequency domain channel matrix $G$ is a diagonal matrix, defining its diagonal entries as $G[k], k=1,2, \cdots, N$. Then we can rewrite Eq.11 in individual symbol:

$$
\hat{S}_{\text {OFDM }}[k]=G^{-1}[k] y[k], \quad k=1,2, \cdots, N
$$

In case of the S-OFDM, for the estimation of individual symbol, according to the process of code spreading, we can easily get:

$$
\hat{S}_{\text {SOFDM }}[k]=\mathrm{C}_{k}^{H} \hat{S}_{\text {OFDM }}, k=1,2, \ldots, N
$$

Note that $\mathrm{C}_{k}$ is the $k$ th volume of the spread matrix $C$. In other words, the $\hat{S}_{\text {OFDM }}$ is a spread symbol vector which is de-spread into $\hat{S}_{\text {SOFDM }}[k]$ by the vector $\mathrm{C}_{k}^{H}$.

\section{B. MMSE}

As weak subcarriers are amplified with high gain in ZF, it introduces a high noise level. For this reason ZF detector is easily influenced by noise. So MMSE is introduced considering the noise variance. It is based on the criterion that finding the matrix $T$ to minimize the mean square error:

$$
\arg \min _{w} E\left\{\left(s-T^{H} y\right)^{2}\right\}
$$

The solution is given as[6]:

$$
T=\left(G C C^{H} G^{H}+\sigma_{v}^{2} I_{N}\right)^{-1} G C
$$

So the resulted MMSE symbols will be as follows:

$$
\hat{s}[n]=T^{H} \mathrm{y}[n]
$$

$$
\hat{s}=C^{H} G^{H}\left(G C C^{H} G^{H}+\sigma_{v}^{2} I_{N}\right)^{-1} \mathrm{y}
$$

For the conventional OFDM with no spread, $C=I_{N}$, the detected symbol is:

$$
\hat{S}_{\text {OFDM }}=G^{H}\left(G G^{H}+\sigma_{v}^{2} I_{N}\right)^{-1} \mathrm{y}
$$

Define the diagonal entries of $G$ as $G[k], k=1,2, \cdots, N$. Then we can rewrite Eq.18 in symbol:

$$
\hat{S}_{\text {OFDM }}[k]=\frac{G^{*}[k] y[k]}{|G[k]|^{2}+\sigma_{v}^{2}}, k=1,2, \cdots, N
$$

And for S-OFDM, generally the spread matrix is unitary matrix, $C C^{H}=I_{N}$, then the detected symbol can be expressed:

$$
\hat{S}_{\text {SOFDM }}=\mathrm{C}^{H} G^{H}\left(G G^{H}+\sigma_{v}^{2} I_{N}\right)^{-1} \mathrm{y}
$$

For the estimation of individual symbols, based on the conventional OFDM, we have:

$$
\hat{S}_{\text {SOFDM }}[k]=\mathrm{C}_{k}^{H} \hat{S}_{\text {OFDM }}, k=1,2, \ldots, N
$$


Note that $\mathrm{C}_{k}$ is the $k$ th volume of the spread matrix $C$. It is the same as ZF algorithm, $\hat{S}_{\text {SOFDM }}[k]$ can be de-spread from $\hat{S}_{\text {OFDM }}$ by the vector $\mathrm{C}_{k}^{H}$.

\section{Proposed ZF Detection}

Comparing two detectors, we find that the ZF detector can resist the decline of Rayleigh distributed channel entirely, but ignore the impact of amplifying noise when channel power is very low which will cause terrible BER performance. The MMSE detector takes the noise into account, but the MMSE matrix cannot accurately eliminate the channel impact and require estimate the variance of noise. So, our purpose is to do some improvement based on ZF detector, to suppress the noise under accurate channel equalization.

From sect.2 we can find that using S-OFDM is to resist the frequency selective attenuation through spreading all symbols across all OFDM subcarriers to solve the degradation of individual subcarrier. And in the receiver, after FFT, every received symbol will be de-spread into all data to recover the original data symbol. But if one subcarrier undergoes sever degradation, then through the ZF detection the noise will be amplified, after de-spread, the big noise is spread into all symbols, so the performance of S-OFDM with ZF detector will be bad.

After OFDM detection, the received signal can be expressed:

$$
\mathrm{x}=G \mathrm{Cs}+F C_{p r} v
$$

Note that $G=F C_{p r} H C_{p} F^{H}$ is the equivalent diagonal frequency-domain channel, then before de-spread, the result of ZF detector is:

$$
\begin{aligned}
\hat{\mathrm{S}}_{\text {OFDM }} & =(G)^{-1} \mathrm{x} \\
& =\mathrm{Cs}+\frac{F C_{p r} v}{G}
\end{aligned}
$$

Thus, in S-OFDM, if the value of $G$ is small, then the equivalent frequency-domain noise $F C_{p r} v / G$ will be large, this large noise is spread into other symbols through multiplying $C^{H}$.

We know that in the S-OFDM system, a missed symbol could be recovered through other symbols during the de-spread process. So during the ZF detection, if we find one subcarrier is deeply degradation, we can abandon it together with its large noise caused by ZF detector. Then in the process of de-spread, we can recover this subcarrier with little error, meanwhile wipe off the influence of large noise. The specific method is to set the data to zero(the mean of transmitted data) whose channel degradation value is lower than a threshold L:

$$
\hat{\mathrm{S}}_{\text {OFDM }}[k]=\left\{\begin{array}{cc}
G^{-1}[k] \mathrm{x}[k] & |G[k]|^{2} \geq L \\
0 & |G[k]|^{2}<L
\end{array} \quad k=1,2, \cdots, N\right.
$$

Then we de-spread the getting data:

$$
\hat{\mathrm{S}}_{\text {SOFDM }}[k]=\mathrm{C}_{k}^{H} s_{\text {OFDM }}, k=1,2, \ldots, N
$$

From the MATLAB simulation in the next section, we can know that for multi-path underwater acoustic channel, the proposed ZF detector can efficiently reduce BER with a proper threshold.

\section{Channel Model and Simulation Results}

In this section, we will show the performance of S-OFDM with different detectors using BER performance in underwater channel. Channel characteristic plays an important role in designing the scheme. Underwater test shows that the channel often exhibits poor than a Rayleigh distributed channel modeling due to the sever signal fading and complicated noise from different sources. The change of position and time also has significant impact on the channel property. Less deep acoustic channel has been modeled as a $L$ path Rayleigh channel, the $L$ is correlated to the maximal delay[1,2,3]. Additive white Gaussian noise (AWGN) for the system is combined at the receiver. The channel response can be expressed:

$$
h(t, \tau)=\sum_{i=1}^{M} a_{i}(t) \delta\left(t-\tau_{i}\right)
$$

Where $M$ is the number of rays using in the multipath channel model. The $a_{i}(t)$ and $\tau_{i}$ are channel magnitudes and propagation delay of $k$ th path. A detailed study can be found in [3].

In these simulations, we choose QPSK modulation, and 128 subcarriers in OFDM. Where $L=15$ for the maximum delay is 15 times of one symbol duration. And the channel is assumed to be steady during an OFDM block whose energy is normalized to 1 . Meanwhile we assumed that the receiver knows perfect channel information. And for ISI, we used a cyclic prefix (CP) whose length is equal to the maximum delay to maintain the orthogonality of subcarriers while eliminating ISI. The bit error rate (BER) for OFDM and S-OFDM with different existing detectors was simulated in Figure 2. 


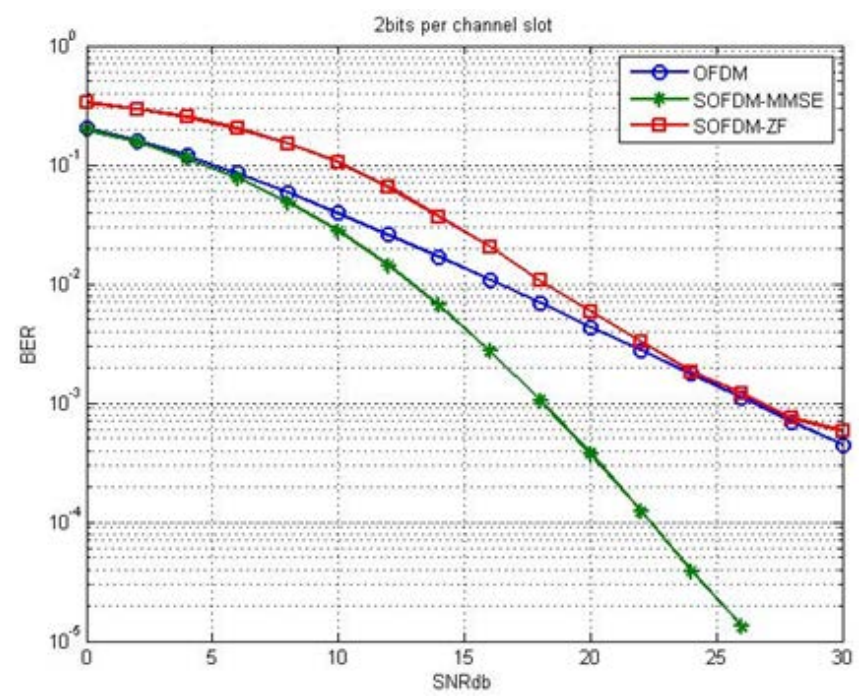

Figure 2. BER results for OFDM and S-OFDM with MMSE and ZF detector

Note that the S-OFDM with MMSE detector shows better performance than conventional OFDM owing to its immunity to the channel nulls and resistance to the noise spread. The gain is about $8 \mathrm{~dB}$ when BER is $10^{-3}$. On the contrary, for the reason of spreading the big noise to entire spectrum, S-OFDM with ZF detector shows even bad to ordinary OFDM.

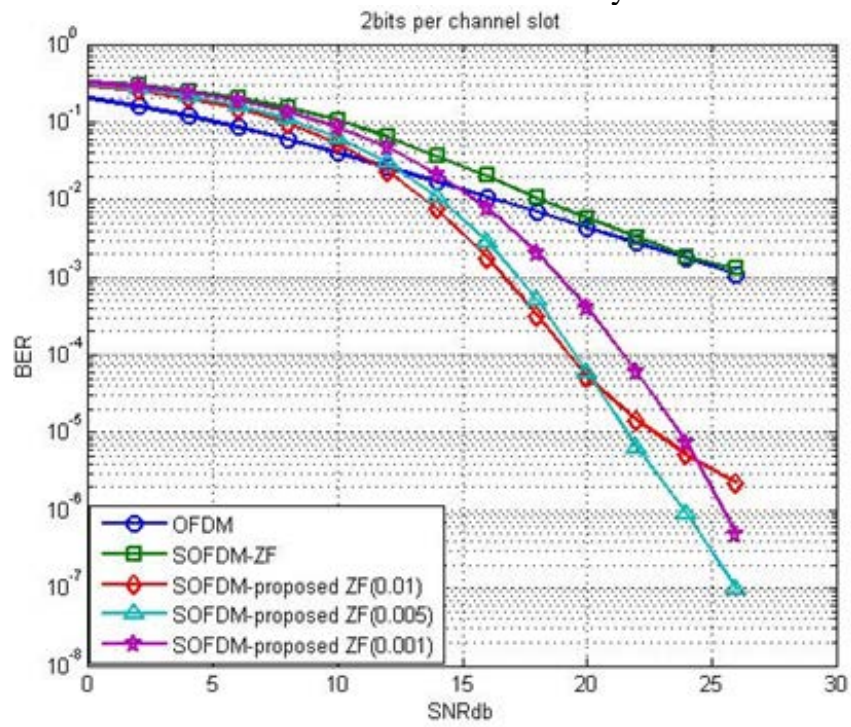

Figure 3. BER results for ZF-S-OFDM and proposed ZF-S-OFDM with some proper thresholds

Note that comparing the proposed ZF detector to conventional ZF detector, we can find there is an obvious gain in BER. It means that the abandon of deeply degraded subcarriers works to prevent the spread of big noise. The gain is around $9 \mathrm{~dB}$ when BER is $10^{-3}$.

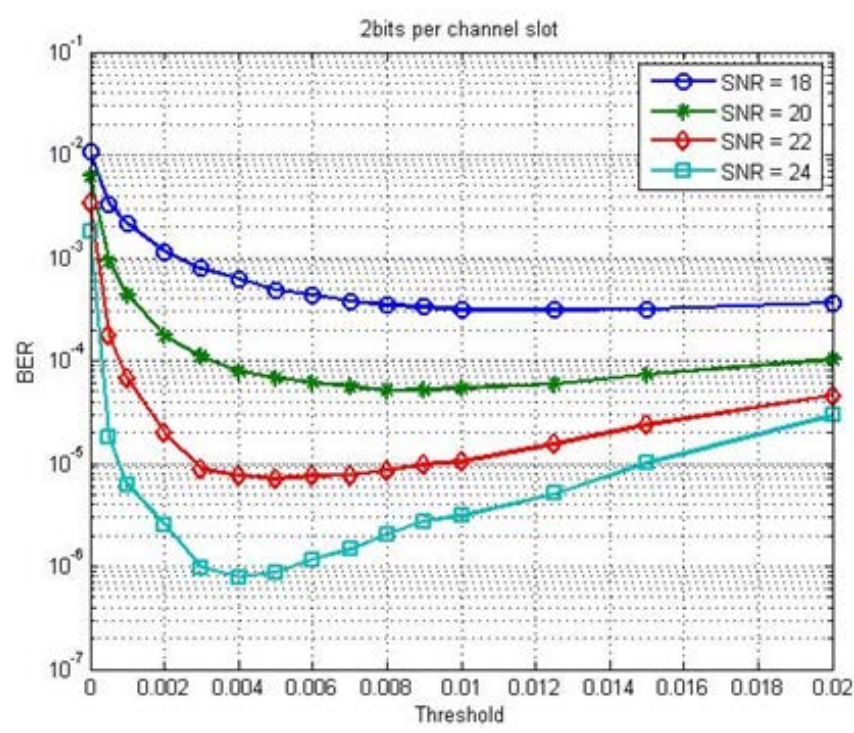

Figure 4. BER results of proposed ZF-S-OFDM for threshold ranging from 0 to 0.02 in different SNR

Then we want to find a proper threshold for this simulation condition. So we test the BER of proposed ZF-OFDM for different threshold in Fig.4. Note that the best threshold is ranging with SNR. In general, when the threshold is around 0.005, the BER for all different SNR approaches their minimum value, so we consider the most proper threshold is 0.005 in this simulation condition.

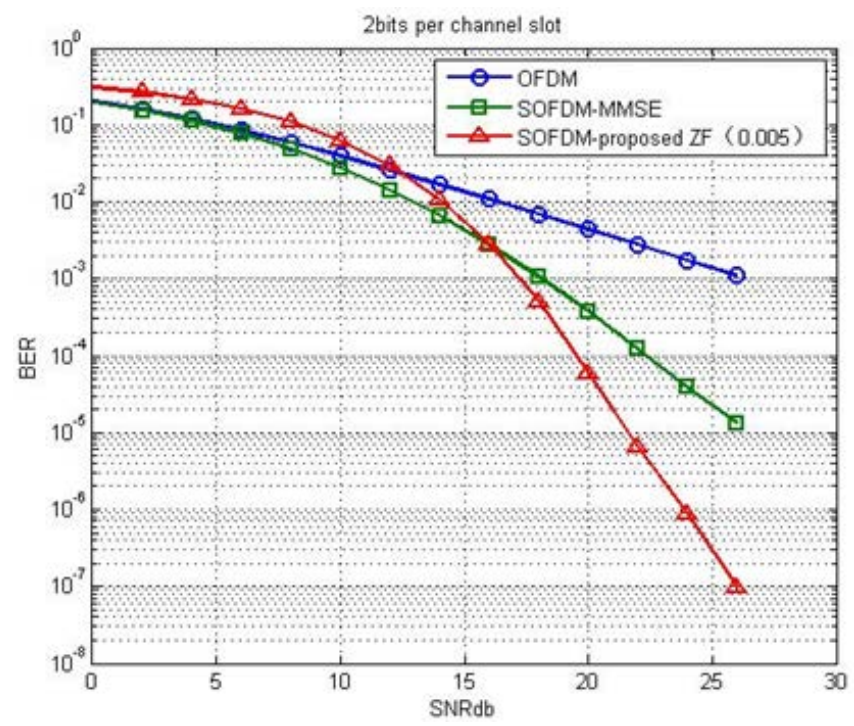

Figure 5. BER results for MMSE-S-OFDM and proposed ZF-S-OFDM with best threshold

Compared with MMSE detector, the proposed ZF detector shows even better in high SNR, it has a gain around $5 \mathrm{~dB}$ to MMSE detector when SNR is $10^{-5}$, which means that proposed ZF detector synthesizes the merit of ZF and MMSE detector and has better adaptation in underwater channel. 


\section{CONCLUSION}

S-OFDM system has ability to ensure multipath resilience without any data losses or increasing in the average transmitted energy. By analyzing the weakness of MMSE and $\mathrm{ZF}$ detectors, an improved threshold based $\mathrm{ZF}$ detector is proposed to prevent the spread of noise and resist the degradation of channel entirely. Simulation results demonstrated that the performance of proposed ZF detector has an obvious improvement to the $\mathrm{ZF}$ detector. Compared to the MMSE detector, proposed ZF detector also performs better in high SNR without the need of estimating noise variance.

\section{REFERENCES}

[1] Stojanovic, M., \& Preisig, J. Underwater acoustic communication channels: Propagation models and statistical characterization. IEEE Communications Magazine, 47(1), 84-89. (2009).

[2] Domingo, M. C. Overview of channel models for underwater wireless communication networks. Physical Communication, 1(3), 163-182. (2008).

[3] Zielinski, A., Yoon, Y. H., \& Wu, L. Performance analysis of digital acoustic communication in a shallow water channel. IEEE Journal of Oceanic Engineering, 20(4), 293-299. (1995).
[4] Zhendao Wang, Giannakis G. B., "Wireless multicarrier communications," Signal Processing Magazine, IEEE, vol.17,no.3,pp.29,48, May 2000.

[5] Hamada Esmaiel, Danchi Jiang. Review Article: Multicarrier Communication for Underwater Acoustic Channel. Int. J. Communications, Network and System Sciences, 2013, 6, 361-376.

[6] Ahmed M. S., Boussakta S., Sharif B. S., Tsimenidis, C. C., “OFDM Based on Low Complexity Transform to Increase Multipath Resilience and Recude PAPR,” Signal Processing, IEEE Transactions on , vol.59, no.12, pp.5994,6007, Dec. 2011.

[7] Al-Mahmoud, M., \& Zoltowski, M.D. Performance evaluation of code-spread OFDM using vandermonde spreading. In IEEE radio and wireless symposium, pp. 320-323. (2009).

[8] Dlugaszewski, Z., \& Wesolowski, K. WHT/OFDM-an improved OFDM transmission method for selective fading channels. In symposium on communications and vehicular technology, 2000. SCVT-200, pp. 144-149.

[9] Kumar, P., \& Kumar, P. A comparative study of spread OFDM with transmit diversity for underwater acoustic communications. Wireless Pers Commun DOI 10.1007/s11277-015-2380-8. (2015).

[10] H. Ding, J. A. Neasham, S. Boussakta and C. C. Tsimenidis, "Performance Evaluation of T-transform based OFDM in Underwater Acoustic Communications,” OCEANS 2015 - Genova,Genoa,2015, pp.1-7.

[11] Ali Elghariani and Michael D. Zoltowski "Partial Spread OFDM" Wireless Sensing. Proc. of SPIE Vol. 8404, 84040B $\cdot 2012$ 